Revue des patrimoines

$21 \mid 2013$

De l'art de bâtir aux champs à la ferme moderne

EDITORIAL

\title{
De l'art de bâtir aux champs à la ferme moderne
}

Jean-Philippe Garric

\section{(2) OpenEdition}

Journals

Édition électronique

URL : http://journals.openedition.org/insitu/10467

DOI : 10.4000/insitu. 10467

ISSN : 1630-7305

Éditeur

Ministère de la Culture

Référence électronique

Jean-Philippe Garric, " De l'art de bâtir aux champs à la ferme moderne », In Situ [En ligne], 21 | 2013, mis en ligne le 12 juillet 2013, consulté le 22 septembre 2020. URL : http://journals.openedition.org/ insitu/10467 ; DOI : https://doi.org/10.4000/insitu.10467

Ce document a été généré automatiquement le 22 septembre 2020.

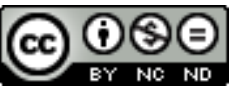

In Situ Revues des patrimoines est mis à disposition selon les termes de la licence Creative Commons Attribution - Pas d'Utilisation Commerciale - Pas de Modification 4.0 International. 


\title{
De l'art de bâtir aux champs à la ferme moderne
}

\author{
Jean-Philippe Garric
}

1 Ce numéro de la revue In Situ est composé des textes issus des deux colloques intitulés "L'art de bâtir aux champs » et "Les campagnes européennes et les territoires coloniaux, entre tradition et innovation", organisés en 2010 et 2012 par la Direction générale des patrimoines et l'Institut national d'histoire de l'art, sous la responsabilité scientifique de Jean-Philippe Garric, Emilie d'Orgeix, Isabelle Roland, Bernard Toulier et Pascal Liévaux.

2 Les textes ici rassemblés montrent ce que l'on pouvait bien pressentir, mais qui prend une tournure plus précise et souvent plus poignante au spectacle des projets, des ruines, des reconstructions et, finalement, de l'impact souvent violent des édifices agricoles contemporains sur le paysage. Après un long $\mathrm{XIX}^{\mathrm{e}}$ siècle optimiste, vecteur de modernisation, d'industrialisation et de développement, les campagnes européennes, même si elles eurent aussi leur part des espoirs et des ambitions qui ont cru y porter remède, ne furent épargnées ni par les violences, ni par les entêtements idéologiques qui déchirèrent le $\mathrm{xx}^{\mathrm{e}}$ siècle, ni par l'obsolescence des anciennes formes d'organisation spatiale inhérentes à la déterritorialisation libérale des pratiques.

\section{Quel objet, quelle approche?}

3 « Architecture rurale»; « architecture rustique»; "constructions rurales »; "constructions agricoles», sont quelques-unes des dénominations les plus communes - en partie seulement synonymes -, qui désignent le domaine aux contours incertains, aux marges de l'architecture, sur lequel portent ces recherches. Mais cette situation excentrée n'affaiblit ni les enseignements, ni la valeur heuristique des cas étudiés, qui sont au contraire aussi révélateurs des grandes mutations de la discipline que des édifices mieux connus et plus réputés, jouissant d'une position centrale. Ils illustrent les évolutions de l'architecture à la période contemporaine : son ouverture à de nouveaux 
programmes, sa démocratisation en direction de nouvelles catégories de commanditaires et d'usagers, le développement du rôle de l'imprimé, ou encore l'industrialisation des matériaux, de leur transport, de leurs procédés de mise en œuvre, qui supplantent les ressources et les pratiques locales. Mais cette frontière, qu'on peut envisager dans une perspective enthousiaste - au sens de la conquête -, dans les phases de rapide expansion qui caractérisent le siècle de l'industrie, prend en revanche, après la Seconde Guerre mondiale, le sens d'un délaissé, d'un paysage à l'abandon.

4 À l'initiative de cette publication, la réunion d'une approche de terrain à visée patrimoniale, centrale dans les travaux des équipes de chercheurs de l'Inventaire et du ministère de la Culture, et d'une étude des sources imprimées, menée par l'Institut national d'histoire de l'art dans le cadre de la «Bibliographie du livre d'architecture français (1512-1914)», est un choix qui reflète la nature double d'un objet, lui-même partagé entre les cultures constructives et architecturales locales, les savoirs et savoirfaire transmis oralement, et l'intrusion progressive de nouveaux principes et de modèles nationaux - voire internationaux -, diffusés par l'imprimé. Croiser ainsi un regard aguerri à l'archéologie du bâti et un autre relevant de l'histoire culturelle, revient à prendre l'architecture rurale dans une perspective qui peut s'appliquer plus largement à toute l'architecture de la période contemporaine.

\section{De la floraison théorique au déclin}

Figure 1

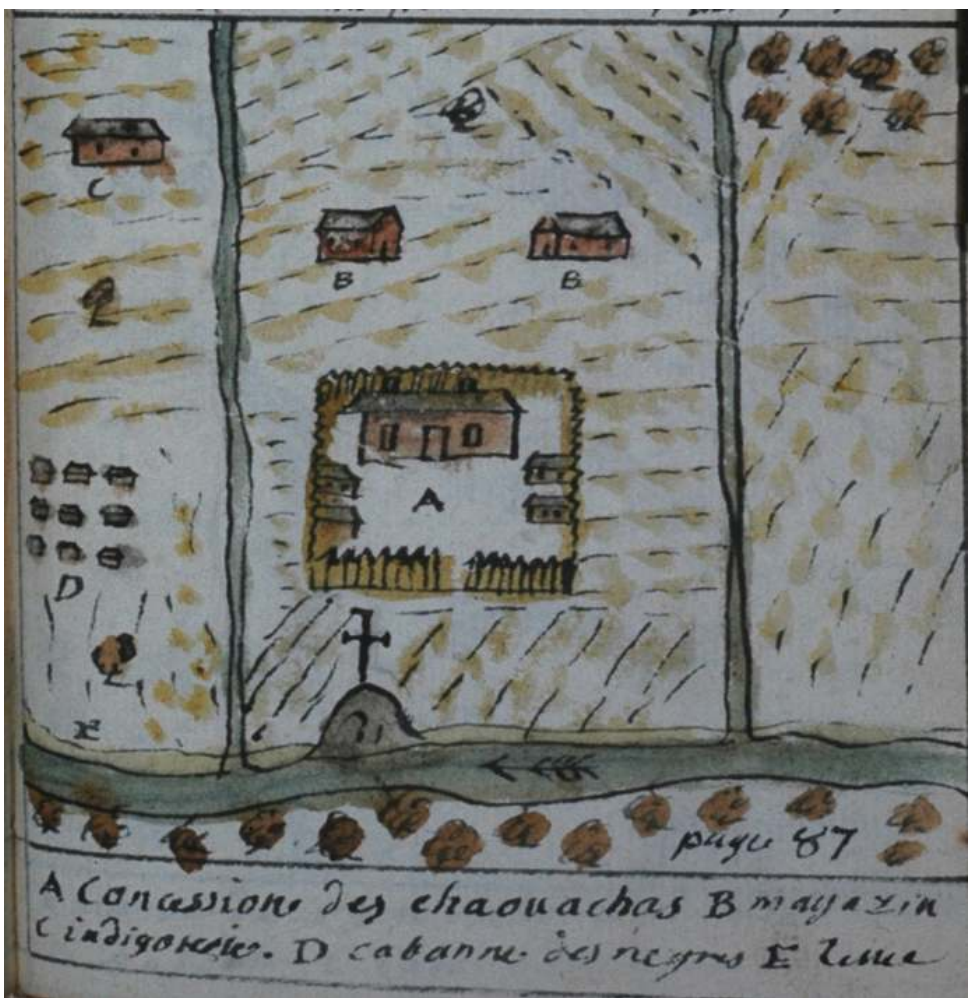

Benjamin Dumont de Montigny, concession des Chaouachas, Louisiane, Etats-Unis, ca1730

(c) BNF, Arsenal, ms 3459, p. 148. 
En France, sous l'Ancien Régime, les premiers progrès des exploitations et des constructions agricoles s'inscrivent dans un contexte aristocratique, comme le château de Burk à Ambès, étudié ici par Laurence Chevallier, ce qui n'exclut pas la recherche du rendement financier, dont témoignent les fermes modèles de Louisiane (fig. $\left.\mathbf{n}^{\circ} \mathbf{1}\right)$. Mais les livres sur l'architecture rurale restent rares ; celui du chevalier de Préfontaine, présenté par Émilie d'Orgeix et Céline Frémaux, fait plutôt figure d'exception. Et c'est seulement à partir de la Révolution et de l'Empire qu'une floraison de publications - à l'origine desquelles la contribution féconde et singulière de François Cointeraux occupe une position centrale - marque le début d'une époque nouvelle (fig. $\mathbf{n}^{\circ} \mathbf{2}$ ).

Figure 2

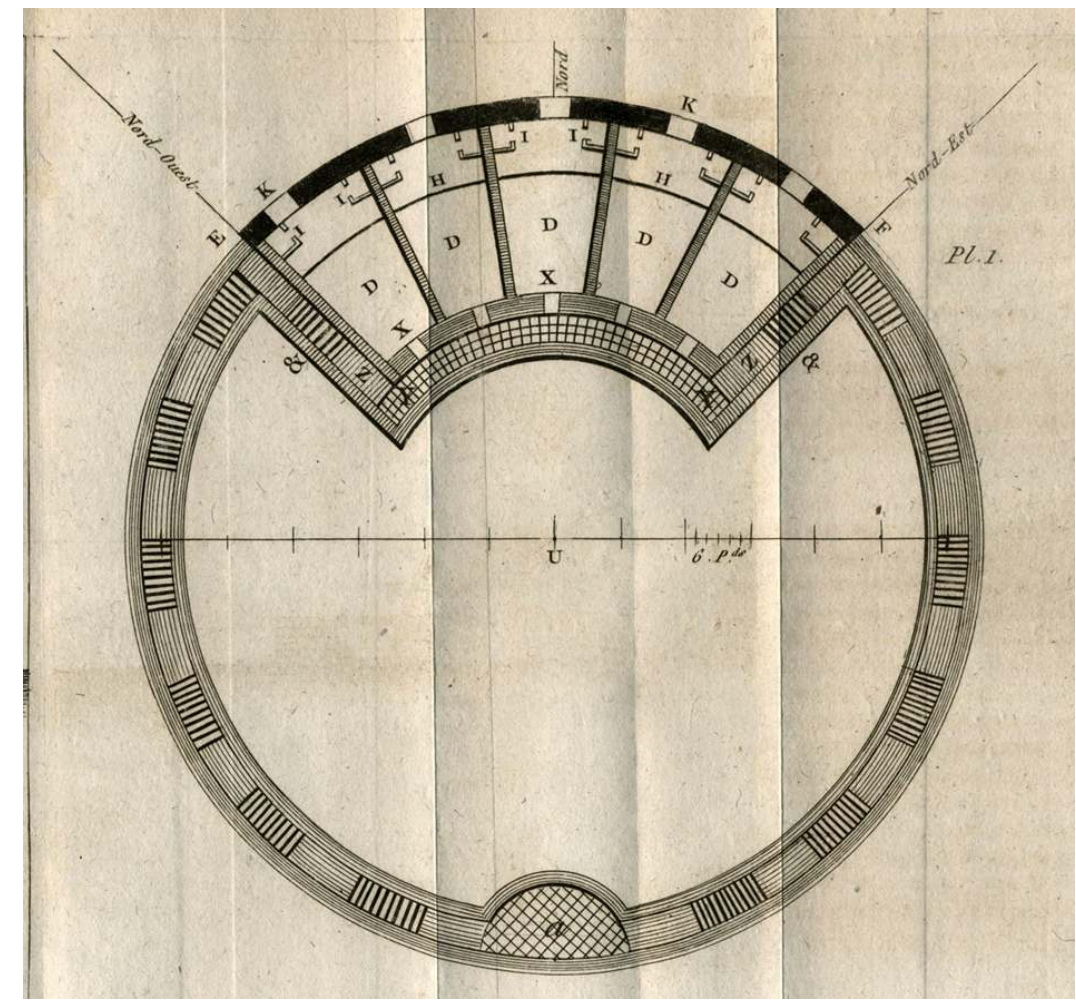

François Cointeraux. Plan d'une faisanderie, Nouvelles dispositions de faisanderies, 1805.

Repro. Garric, Jean-Philippe. (c) Jean-Philippe Garric.

6 Ce mouvement, dont Véronique Samuel-Gohin montre qu'il connaît un équivalent OutreRhin, permet la diffusion de propositions spéculatives, ou de modèles de bâtiments ou d'aménagements, rendant compte d'expérimentations, comme celles que Morel-Vindé réalise à ses frais dans son propre domaine. Les auteurs sont des architectes, des ingénieurs ou de simples "propriétaires " et les thèmes abordés, ou les visées de ces publications, varient de la célébration d'un "art utile» au souci d'économie ou de rationalisation des bâtiments de la ferme et des processus d'exploitation. Mais ces ambitions architecturales, étendues à des constructions le plus souvent réduites à des visées utilitaires, révèlent aussi pourtant un souci de représentation sociale et recèlent des pratiques créatives (fig. $\mathbf{n}^{\circ} \mathbf{3}$ ). La ferme est un microcosme propice à l'exercice privé du pouvoir - notamment du pouvoir d'entreprendre -, voire à la construction "d'utopies domestiques", en même temps qu'elle participe d'un réseau de sociabilité et d'échanges, contribuant parfois, comme pour les chais du bordelais, à bâtir une image de marque. 
Figure 3

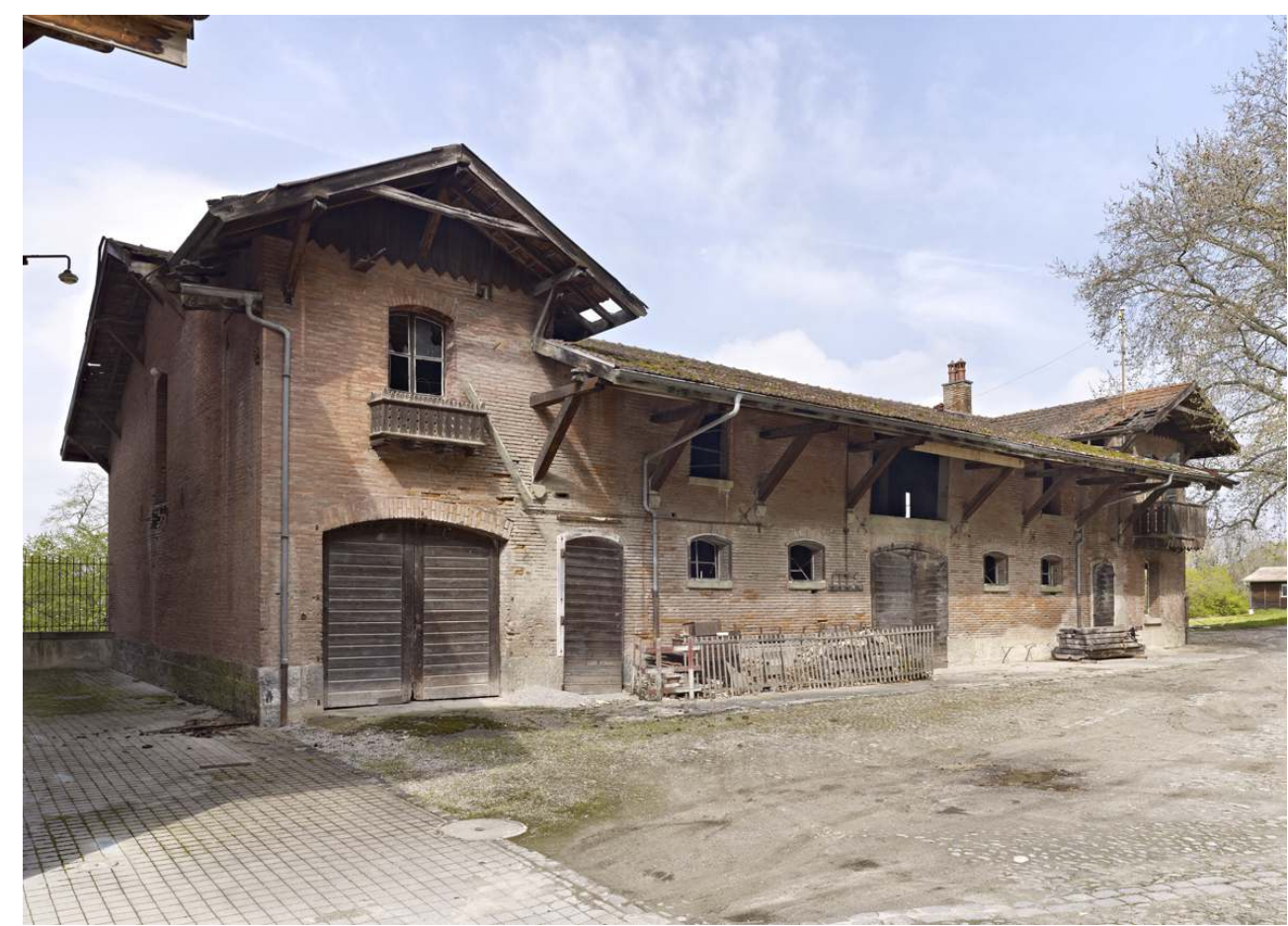

Ferme de Beau-Cèdre, Jouxtens-Mézery, Suisse (canton de Vaud) : la vacherie, vue de la façade principale.

Phot. R. Gindroz, 2012. (c) R. Gindroz.

$7 \quad \mathrm{Au} \mathrm{Xx} \mathrm{X}^{\mathrm{e}}$ siècle, le transfert de population des campagnes européennes vers les villes, déjà largement engagé au siècle précédent, s'amplifie, confirmant la prééminence de la civilisation urbaine. La ville s'y affirme, au détriment de l'espace rural, comme le lieu de convergence des enjeux contemporains, des innovations et des dynamiques culturelles. Sa croissance concentre l'attention des architectes et des urbanistes et, par contrecoup, celle des historiens. Pourtant, si l'architecture du $\mathrm{xx}^{\mathrm{e}}$ siècle est d'abord urbaine, le monde rural fait lui aussi l'objet de multiples ambitions et entreprises privées ou publiques de modernisation. Certaines poursuivent les réformes développées au XIX ${ }^{\mathrm{e}}$ siècle, dont elles partagent les visées planificatrices, industrielles, productivistes, hygiéniques et réformatrices - c'est le cas du domaine Renault d'Herqueville. D'autres, qui entendent changer le cadre de vie et de production, sont planifiées au niveau national, devenant dès lors des lieux privilégiés d'expression des grandes idéologies politiques: un mouvement qui s'accompagne d'une importante production théorique et de propagande. Au capitalisme agricole succèdent de vastes projets collectivistes ou communautaires, socialistes ou nationalistes, donnant lieu à des programmes à grande échelle d'aménagement du territoire et de transformation de l'environnement bâti.

8 Les conflits internationaux, le développement de la grande industrie et l'expansion coloniale bouleversent la nature et le périmètre du territoire agricole des puissances européennes. L'enjeu des reconstructions et le déplacement des modèles, moteur d'inventions et d'adaptations, exacerbent les prises de conscience et les revendications régionalistes. Le progrès des techniques et la diffusion des nouveaux matériaux, permettant des réalisations plus rapides et plus ambitieuses, se substituent aux pratiques traditionnelles, jusqu'à produire des réactions (fig. $\mathbf{n}^{\circ} \mathbf{4}$ ). 
Figure 4

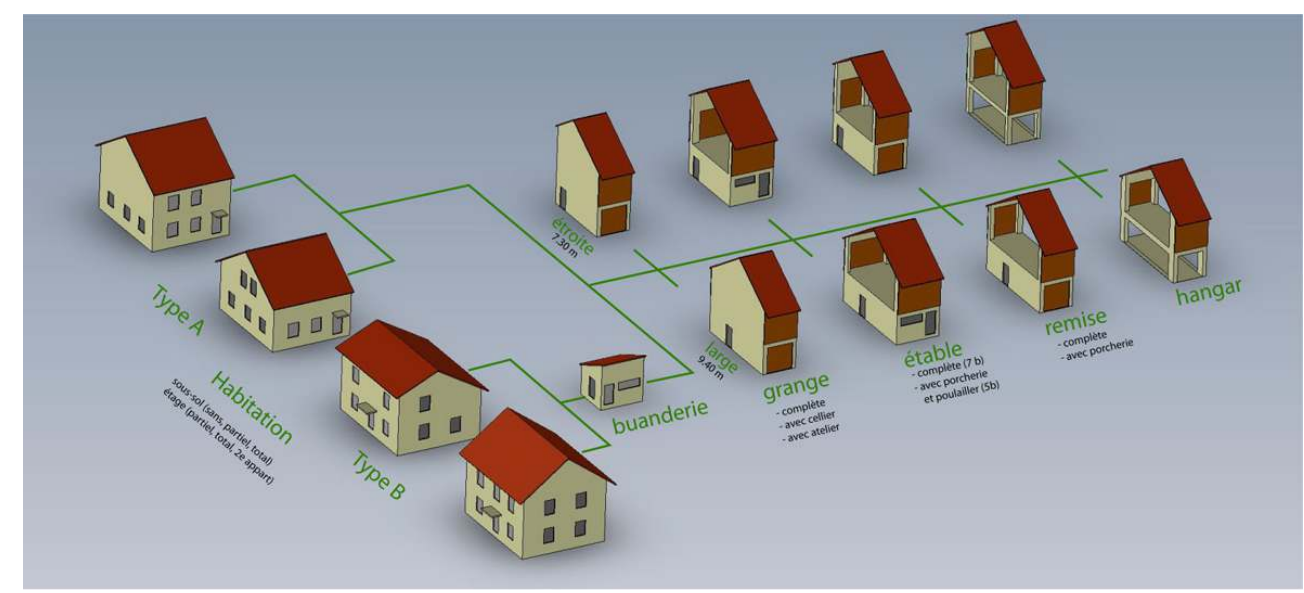

Dessin des différents corps de bâtiments pouvant composer une ferme de l'OP 24, vallée de la Meurthe, Vosges.

Dessin Henry, Jean-Yves. Base Mémoire : IVR41_20128812160NUDA. @ Région Lorraine-Inventaire général.

\section{La désurbanisation comme remède}

Deux textes, dans leur différence et leurs similitudes, éclairent l'actualité de la thématique d'un jour plus cru et inquiétant que celui des images d'Épinal de l'agriculture urbaine, ou de la ville fertile, qui accompagnèrent notamment certaines des réponses à la consultation du Grand Paris. Dans la parenté asymétrique des projets proposés par Leberecht Migge à la république de Weimar et de ceux poursuivis par le régime fasciste avec la bonification de la Sicile intérieure, se dessine une même question, aussi ancienne que la théorie d'architecture rurale, telle qu'elle émergea dans la France et l'Europe des Lumières, celle de la rédemption des classes laborieuses par la terre et de leur désurbanisation.

Car, dans les deux cas, l'enjeu, s'il était d'abord celui-là, n'était pas simplement celui de la création d'une agriculture vivrière devant contribuer à l'autosubsistance, voire à l'autarcie, dans une période de crise économique majeure, il visait aussi, dans une perspective rousseauiste, à abstraire l'individu du maelström urbain, des brouillards industriels mortifères, de l'énervement provoqué par la surexcitation des sens dans la grande métropole machiniste et, finalement, de la corruption morale qu'elle recèle.

11 Trouver dans la colonisation intérieure et dans le retour aux champs plus ou moins volontaire ou contraint des masses populaires une solution à la crise du capitalisme et au chômage de masse ne s'accompagnait pas dans les deux cas des mêmes ambitions. Dans l'un, on parle d'un réformisme social et du paysage fertile d'une agriculture périurbaine, dans l'autre d'une révolution sociale placée sous la bannière néoclassique du "paysan soldat" auquel on paierait le prix du sang par la distribution de la terre aux combattants dans une "Arcadie fasciste", comme à la même époque le slogan "d'ordre nouveau" accompagne la mise en œuvre des fermes allemandes de Marckolsheim, et comme, plus tard, la notion "d'homme nouveau" tente de justifier les crimes contre la paysannerie et contre les villages roumains de Nicolaus Ceausescu. 
tes ces histoires souvent brutales et qui se terminent rarement bien donnent quelques raisons d'hésiter : doit-on regarder ces propositions et ces tentatives comme des modèles à méditer pour faire face aux difficultés du présent, ou comme des précédents pour le moins inquiétants? Les ambitions disproportionnées des palais agricoles technocratiques et modernistes de l'Estonie soviétique, s'ils réjouissent sans aucun doute l'amateur de plastique architecturale par le jeu splendide de leurs volumes dans la lumière du Nord, n'en furent pas moins un autre genre d'impasse (fig. $\mathbf{n}^{\circ} 5$ ). Le fait que les reconstructions allemandes en Alsace et en Lorraine puissent apparaître en comparaison comme des menées à la fois raisonnables et pérennes n'est pas de nature à accroître notre enthousiasme.

Figure 5

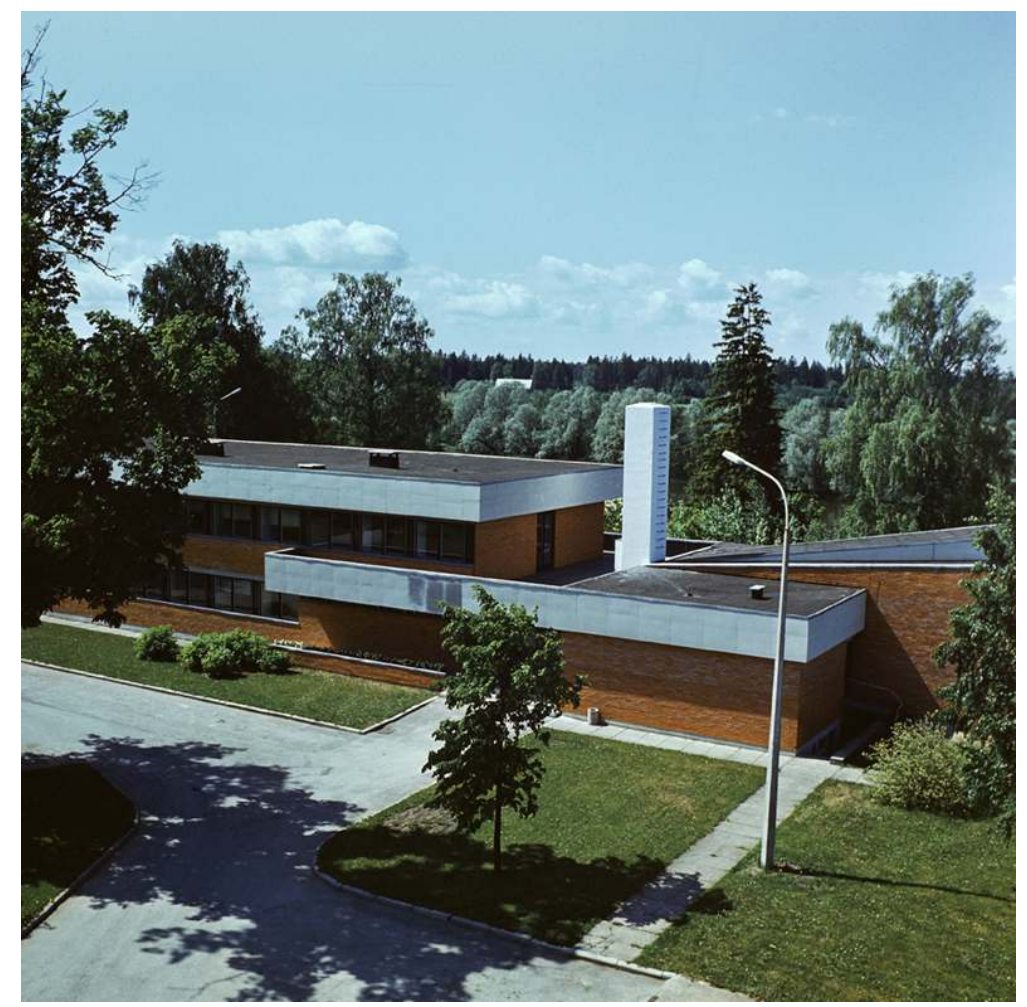

Architecte Valve Pormeister. Bâtiment principal du centre expérimental d'élevage avicole de Kurtna, district de Harju, Estonie, 1965-1966.

Phot. Taska, A. à la fin des années 1970. (A) Archives cinématographiques d'Estonie.

\section{Échecs}

Car ces récits croisés autour de la Ferme moderne s'ils nous présentent des objets bâtis ou non, qui conservent a postériori leur pouvoir de séduction et leur puissance inspiratrice, sont aussi le plus souvent des histoires d'échecs et d'espoirs déçus, qui peuvent se lire dans leur ensemble comme une faillite globale pour les architectes, à prendre toute la part qu'ils ambitionnaient à la construction et à l'aménagement du monde agricole et des paysages ruraux. Une malédiction à laquelle le village du Bosquel ne fait pas entièrement défaut. L'intelligence technologique et typologique et la finesse de la réflexion architecturale, ont permis à ses architectes, émules de Le Corbusier, d'inventer presque à 
eux seuls la voie d'une modernité différente, débouchant non pas sur l'unité d'habitation corbuséenne en rase campagne, plutôt menaçante, qu'avait pensé leur mentor, mais sur une réinvention du village qui anticipait de plus d'une décennie la révision des conceptions urbaines du mouvement moderne qui accompagna la période postérieure à l'institution des Congrès internationaux d'architecture moderne. Mais tout cela n'a pas empêché ce prototype ou ce modèle de manquer la véritable modernisation du monde agricole, celle de la mécanisation et du changement d'échelle. Même le soin pris par les architectes à dialoguer avec les futurs usagers et leur tentative d'inventer une forme de participation dans la conception du projet n'ont pas su éviter cette impasse.

Cet échec est aussi celui de Le Corbusier, qui ne fut pas prié de tester grandeur nature ses propositions dans ce domaine. Mais c'est donc, plus globalement, après 1950, celui des architectes qui sont essentiellement écartés de la construction des édifices agricoles, désormais considérée au plan réglementaire, ainsi que le montre Hervé Cividino, comme relevant de l'équipement technique et non plus de l'immobilier; le hangar à tout faire qu'il présente apparaissant comme le symbole le plus manifeste en même temps que l'agent de ce basculement. Comme le souligne d'ailleurs Daniel Le Couédic dans la préface de l'ouvrage de Hervé Cividino ${ }^{1}$, tout se passe comme si in fine, les architectes modernistes se trouvaient pris au jeu de leurs propres discours sur l'efficacité, sur l'économie et sur la préfabrication, finissant par en être écartés au moment où ces impératifs s'imposent avec toutes leurs conséquences à la réalité de la production industrialisée de l'architecture rurale.

\section{Urbanisme total}

Peut-être le fil conducteur est-il à rechercher dans l'intitulé corbuséen d'urbanisme total. Ce qu'il désigne et qui peut surprendre en particulier dans cette façon d'assimiler l'aménagement rural à une branche de l'urbanisme et à une dimension d'un système ayant vocation à s'étendre au territoire de façon universelle, c'est le dépassement de l'opposition ville - campagne.

On peut remarquer qu'Étienne Louis Boullée dans son Essai sur l'art, s'il esquissait dès la fin du xvIII siècle un projet architectural à l'échelle d'un pays entier, qu'il proposait de mettre en application en Égypte pour tirer parti des grands monuments antiques, mais aussi, croyait-il, de l'insignifiance des architectures modernes dont on pouvait faire table rase, pensait le monde rural uniquement en creux $^{2}$. Mais cette dissolution du fait urbain par son extension à l'ensemble du territoire, que Françoise Choay a reconnu, l'une des premières, comme une caractéristique déterminante de l'évolution contemporaine de la civilisation urbaine de l'Europe, voulu comme un projet par Le Corbusier, n'est-elle pas finalement advenue moins comme la conséquence de choix concertés que comme la résultante d'une absence de pensée d'ensemble, qui, a défaut d'être totalitaire, s'est bien imposée à nous comme une réalité globale ? Elle renvoie indirectement à la disparition du paysan théorisée par Marx et Engels et à ce double mouvement de ruralisation de l'ouvrier et d'urbanisation des ruraux qui semble opposer les régimes autoritaires réactionnaires et progressistes et qui est à l'arrière de bien des démarches de transformations des modes d'habiter et des établissements humains agricoles observés et étudiés dans les textes qui suivent (fig. $\mathbf{n}^{\circ} \mathbf{6}$ ). 


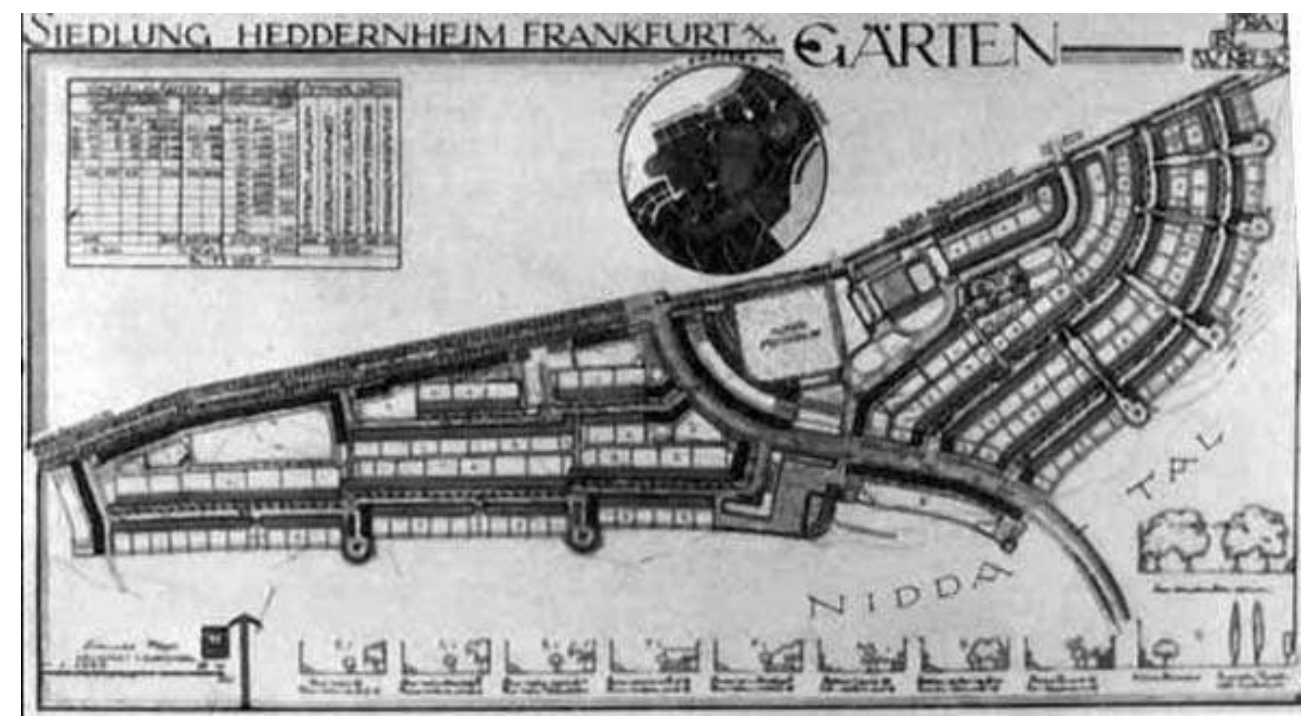

Siedlung Heddernheim, jardins d'agrément et potagers à l'arrière des maisons en rangée, Francfort République fédérale d'Allemagne.

Repro. Jaquand, Corinne. (c) Corinne Jaquand.

\section{Perspectives}

Sans doute a-t-on ainsi le droit de conclure sur une note malgré tout positive, en rapprochant deux perspectives, qu'on jugera peut-être par trop optimistes. Dans les textes ici rassemblés, nous percevons peu l'écho de la parole des ruraux. Comme au XIX siècle, où François Cointeraux pouvait se revendiquer comme le seul architecte issu du monde rural et de la classe populaire, Norbert Bézard apparaît comme l'une des rares figures témoignant de l'aspiration du monde paysan à penser l'avenir de la paysannerie. Cela nous ramène à la première interrogation formulée par Isabelle Roland et qui est revenue périodiquement dans la suite de nos débats, celle du point de vue des occupants, des usagers et pour le dire plus simplement des premiers concernés. J'ai déjà évoqué les efforts de Dufournet et de son équipe pour prendre appui sur un dialogue. Point de vue en amont du projet, point de vue sur la réalité de ces constructions que l'on pense pour eux et qu'on leur impose, cette façon de projeter constamment sur le monde et sur l'espace rural des désirs formulés depuis un point de vue urbain fait écho au beau titre proposé par Pierre Bourdieu à la fin des années 1970, lorsqu'il désignait la paysannerie comme " une classe objet », une classe pour reprendre ses propres termes qui ne parle pas mais «que l'on parle ${ }^{3}$. Si l'opposition ville campagne telle qu'elle a structuré pendant des siècles l'imaginaire européen a cessé d'exister, la paysannerie, en tant que classe pensée et prise en compte par les élites urbaines, est-elle encore une catégorie pertinente? 


\section{NOTES}

1. - Daniel LE COUEDIC dans CIVIDINO Hervé. Architectures agricoles. La modernisation des fermes 1945-1999. Rennes : Presses universitaires de Rennes, 2012, p. 11.

2. - Étienne Louis Boullée, Essai sur l'art, publié par Jean-Marie Pérouse de Montclos. Paris: Hermann, p. 35.

3. - BOURDIEU, Pierre. "Une classe-objet». Actes de la Recherche en Sciences Sociales, $\mathrm{n}^{\circ} 17-18$, novembre 1977, p. 2-5.

\section{AUTEUR}

JEAN-PHILIPPE GARRIC

Conseiller scientifique à l'INHA pour l'histoire de l'architecture jean-philippe.garric@inha.fr 